\title{
DISPERSION OF A SOLUTE IN PERISTALTIC MOTION OF A COUPLE STRESS FLUID THROUGH A POROUS MEDIUM
}

\author{
HABTU ALEMAYEHU AND G. RADHAKRISHNAMACHARYA
}

\begin{abstract}
The paper presents an analytical solution for dispersion of a solute in the peristaltic motion of a couple stress fluid through a porous medium in the presence of both homogeneous and heterogeneous chemical reactions. The average effective dispersion coefficient has been found using Taylor's limiting condition and long wavelength approximation. The effects of various relevant parameters on the average coefficient of dispersion have been studied. The average effective dispersion coefficient increases with permeability parameter but decreases with homogeneous chemical reaction rate parameter, couple stress parameter and heterogeneous reaction rate parameter.
\end{abstract}

\section{Introduction}

Dispersion (or diffusion) of a solute describes the spread of particles through random motion from regions of higher concentration to regions of lower concentration. The dispersion of a solute plays an important role in physiological systems. For example, the knowledge of substances injected in to a blood vessel is useful for many clinical and physiological purposes and also in the distribution of drugs in the human body (Radhakrishnamacharya [21]). The dispersion of soluble matter in fluids has many biological applications especially in the study of blood circulation. Several authors have studied various characteristics of dispersion in fluid dynamical situations which can be applied to biological systems.

Dispersion of a solute in a viscous liquid flowing in a circular pipe under laminar conditions was studied by Taylor [32, 33, 34] and Aris [3]. In all these investigations, it is assumed that the solute does not chemically react in the liquid in which it is dispersed. However, in a wide variety of problems of chemical engineering, diffusion of a solute takes place with simultaneous chemical reaction in situations such as hydrolysis, gas absorption in an agitated tank, esterification (Padma and Ramana Rao [19]). Hence, Gupta and Gupta [10] and Padma and Ramana Rao [19], Ramana Rao and Padma [24, 25] dealt with the effect of chemical reaction on dispersion in Newtonian fluids. Shukla et al. [27], Soundalgekar and Chaturani [29] and

Corresponding author: G. Radhakrishnamacharya.

2010 Mathematics Subject Classification. Primary 421305, 42B08.

Key words and phrases. Dispersion, peristalsis, couple stress fluid, porous medium, chemical reaction. 
Dutta et al. [7] studied dispersion in non-Newtonian fluids by considering only homogeneous first-order chemical reaction in the bulk of the fluid. Chandra and Agarwal [5] considered dispersion in simple microfluid flows taking only homogeneous reaction into consideration. Philip and Chandra [20] also investigated the effects of heterogeneous and homogeneous reactions on the dispersion of a solute in simple microfluid.

Flows through porous media have several applications present in nature: flow in sand beds, in petroleum reservoir rocks, slurries, sedimentation, etc. Many industrial applications involve the modeling of flow through porous media, such as filters, catalyst beds, and packing. Porous materials are used in various engineering devices such as catalytic converters and fuel cells due to advantages in dispersion and chemical reaction by their large contact area. The flow of non-Newtonian fluids through a porous medium under different conditions were studied by Abbasbandy et al. [1], Ashgar et al.[4], Ellahi and Afzal [8], Khan et al. [13] and Khan and Ellahi[14]. Further, several authors have studied the dispersion of a solute through a porous medium under different conditions (Dulal [6], Mehta and Tiwari [15], Al-Nimr and Alkam [2]). Flows through porous media may be relevant in many physiological situations such as the flow of blood in the micro-vessels of the lungs which may be treated as a channel bounded by two thin porous layers (Misra and Ghosh [18]).

Peristalsis is an important physiological mechanism for mixing and transporting fluids, which is generated by a progressive wave of area contraction or expansion moving on the wall of the tube containing fluid. The mechanism is found to be relevant in the gastrointestinal, urinary, reproductive tracts and many other glandular ducts in a living body. Further, peristaltic pumping occurs in many practical applications involving roller and finger pumps. The study of the mechanism of peristaltic transport has been the subject of scientific and engineering research in the last few decades. Several theoretical and experimental studies have been conducted to understand peristaltic transport under different conditions (RamachandraRao and Manoranjan [23]). Fung and Yih [9], Shapiro et al. [26] studied peristaltic transport of Newtonian fluids under different situations.

It is well known that most physiological fluids including blood behave as non-Newtonian fluids. Hence, the study of peristaltic transport of non-Newtonian fluids may help to get better understanding of the biological systems. Several researchers studied peristaltic transport of non-Newtonian fluids (Radhakrishnamacharya [22], Ramachandra Rao and Mishra [23], Misra and Pandey [17] and Hayat et al. [11]).

Couple stress fluids are fluids consisting of rigid, randomly oriented particles suspended in a viscous medium, such as blood, lubricants containing small amount of high polymer additive, electro-rheological fluids and synthetic fluids. The main feature of couple stress fluids is that the stress tensor is anti-symmetric and their accurate flow behavior cannot be predicted by the classical Newtonian theory. Stokes [31] generalized the classical model to 
include the effect of the presence of the couple stresses and this couple stress fluid model has been widely used because of its relative mathematical simplicity compared with other models developed for the couple stress fluid (Islam and Zhou[12]). For couple stress fluids, there have been a number of studies carried out due to its widespread industrial and scientific applications, such as the works of Stokes [31], Srivastava [30], Mekheimer and Abd elmaboud [16] and Sobh [28].

Dispersion of a solute in peristaltic motion of a couple stress fluid through porous medium has not received much attention. Peristalsis and diffusion are very important aspects in biological systems. It is realized that peristalsis may have effect on dispersion of a solute in fluid flow. This, in turn, may help in better absorption of nutrients and drugs in physiological systems. Hence, the study of the interaction of peristalsis with diffusion under different conditions may lead to better understanding of the flow situation in physiological systems. The objective of this paper is to study the dispersion of a solute in peristaltic motion of a couple stress fluid through a porous medium. Using long wavelength approximation and Taylor's approach, closed form solutions have been obtained for the dispersion coefficients for both the cases of homogeneous first-order irreversible chemical reaction and combined first-order homogeneous and heterogeneous chemical reactions. The effects of various relevant parameters on the average effective dispersion coefficient are studied.

\section{Mathematical formulation}

Consider dispersion of a solute in peristaltic flow of couple stress fluid in an infinite uniform channel of width $2 d$. It is assumed that the channel is filled with porous material. Further, the walls of the channel are supposed to be flexible on which traveling sinusoidal waves of long wavelength are imposed. Cartesian coordinate system $(x, y)$ is chosen with the $x$-axis aligned with the center line of the channel. The traveling waves are represented by (Figure 1)

$$
y= \pm h= \pm\left[d+a \sin \frac{2 \pi}{\lambda}(x-c t)\right]
$$

where $a$ is the amplitude, $c$ is the wave speed and $\lambda$ is the wavelength of the peristaltic wave.

Under long wavelength approximation and neglecting body forces and body couples, the equations governing the peristaltic motion of incompressible couple stress fluid through a porous medium for the present problem are given as

$$
\begin{array}{r}
\frac{\partial u}{\partial x}+\frac{\partial v}{\partial y}=0 \\
-\frac{\partial p}{\partial x}+\mu \frac{\partial^{2} u}{\partial y^{2}}-\eta^{\prime} \frac{\partial^{4} u}{\partial y^{4}}-\frac{\mu}{k_{0}} u=0
\end{array}
$$



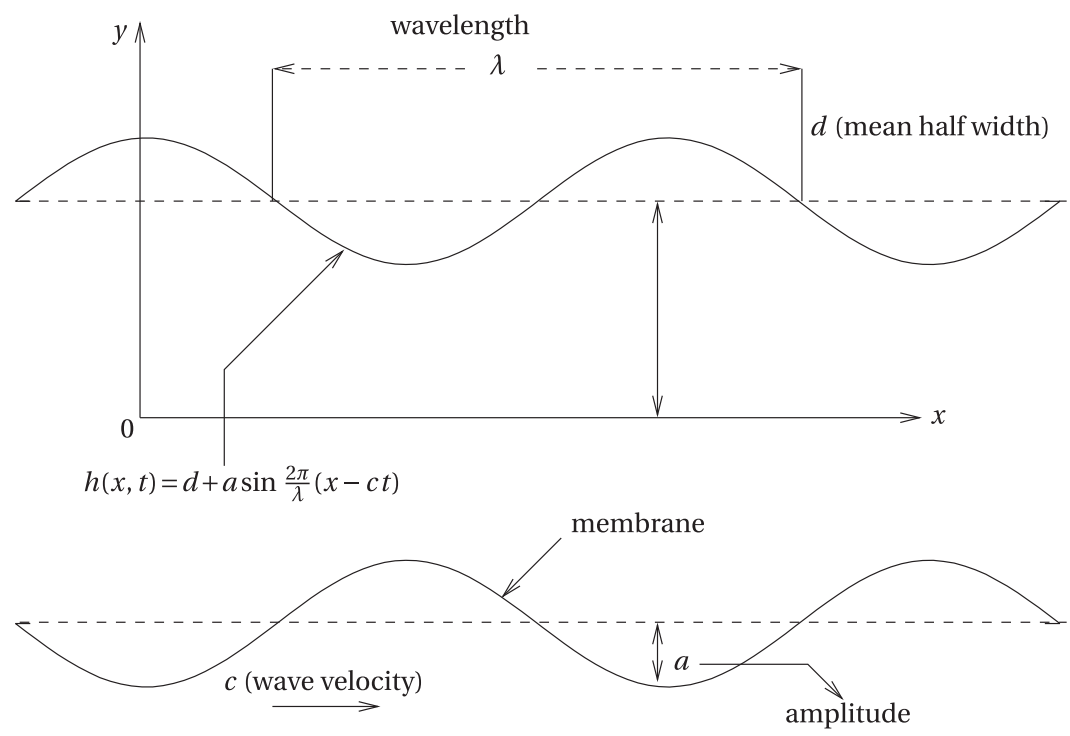

Figure 1: Geometry of the problem.

$$
-\frac{\partial p}{\partial y}=0
$$

where $u(x, y, t)$ and $v(x, y, t)$ are the velocity components in the $x$ and $y$ directions respectively, $P$ is the pressure, $\mu$ is the viscosity coefficient of classical fluid dynamics, $\eta^{\prime}$ is the couple stress fluid viscosity and $k_{0}$ is permeability constant of the medium.

We assume that the walls are inextensible so that only lateral motion takes place and the horizontal displacement of the wall is zero.Thus, the relevant boundary conditions for the velocity are given by

$$
u=0, \quad \frac{\partial^{2} u}{\partial y^{2}}=0 \quad \text { at } \quad y= \pm h
$$

Solving (2.2)-(2.4) under the boundary conditions (2.5), the velocity is given as

$$
u(y)=-\frac{k_{0}}{\mu} \frac{\partial p}{\partial x}\left[S_{2} \cosh \left(m_{1}^{*} y\right)-S_{1} \cosh \left(m_{2}^{*} y\right)+1\right]
$$

where

$$
\begin{aligned}
& m_{1}^{*}=\sqrt{\frac{\mu}{2 \eta^{\prime}}\left(1+\sqrt{1-\frac{4 \eta^{\prime}}{\mu k_{0}}}\right)}, \quad m_{2}^{*}=\sqrt{\frac{\mu}{2 \eta^{\prime}}\left(1-\sqrt{1-\frac{4 \eta^{\prime}}{\mu k_{0}}}\right)}, \\
& S_{1}=\frac{\left(m_{1}^{*}\right)^{2}}{\cosh \left(m_{2}^{*} h\right)\left[\left(m_{1}^{*}\right)^{2}-\left(m_{2}^{*}\right)^{2}\right]}, \quad S_{2}=\frac{\left(m_{2}^{*}\right)^{2}}{\cosh \left(m_{1}^{*} h\right)\left[\left(m_{1}^{*}\right)^{2}-\left(m_{2}^{*}\right)^{2}\right]} .
\end{aligned}
$$

Further, the mean velocity is defined as

$$
\bar{u}=\frac{1}{2 h} \int_{-h}^{+h} u(y) d y
$$


Substituting (2.6) in (2.7) we get,

$$
\bar{u}=-\frac{k_{0}}{\mu} \frac{\partial p}{\partial x}\left[\frac{S_{2}}{m_{1}^{*} h} \sinh \left(m_{1}^{*} h\right)-\frac{S_{1}}{m_{2}^{*} h} \sinh \left(m_{2}^{*} h\right)+1\right] .
$$

If we now consider convection across a plane moving with the mean speed of the flow, then relative to this plane, the fluid velocity is given by

$$
\begin{aligned}
u_{x} & =u-\bar{u} \\
& =-\frac{k_{0}}{\mu} \frac{\partial p}{\partial x}\left[S_{2} \cosh \left(m_{1}^{*} y\right)-S_{1} \cosh \left(m_{2}^{*} y\right)-\frac{S_{2}}{m_{1}^{*} h} \sinh \left(m_{1}^{*} h\right)+\frac{S_{1}}{m_{2}^{*} h} \sinh \left(m_{2}^{*} h\right)\right] .
\end{aligned}
$$

\subsection{Diffusion with a homogeneous first-order chemical reaction}

It is assumed that a solute diffuses and simultaneously undergoes a first order irreversible chemical reaction in peristaltic transport of a couple stress fluid in a channel filled with porous material under isothermal conditions. Hence, the equation for the concentration $C$ of the solute for the present problem satisfies the diffusion equation

$$
\frac{\partial C}{\partial t}+u \frac{\partial C}{\partial x}=D\left(\frac{\partial^{2} C}{\partial x^{2}}+\frac{\partial^{2} C}{\partial y^{2}}\right)-k_{1} C
$$

where $D$ is the molecular diffusion coefficient (assumed to be constant) and $k_{1}$ is the first order reaction rate constant. In deriving (2.10), it is assumed that the solute is present in a small concentration. Using Taylor's assumption (Taylor [32, 33, 34]), i.e., $\frac{\partial^{2} C}{\partial x^{2}}<<\frac{\partial^{2} C}{\partial y^{2}},(2.10)$ becomes

$$
\frac{\partial C}{\partial t}+u \frac{\partial C}{\partial x}=D \frac{\partial^{2} C}{\partial y^{2}}-k_{1} C
$$

For typical values of physiologically relevant parameters of this problem, it is realized that $\bar{u} \approx c$. Using this condition and making use of the following dimensionless quantities

$$
\theta=t / \bar{t}, \bar{t}=\lambda / \bar{u}, \eta=y / d, \quad \xi=(x-\bar{u} t) / \lambda, \quad H=h / d
$$

equation (2.9) reduces to

$$
u_{x}=-\frac{k_{0}}{\mu} \frac{\partial p}{\partial x}\left[S_{2} \cosh \left(m_{1} \eta\right)-S_{1} \cosh \left(m_{2} \eta\right)-\frac{S_{2} \sinh \left(m_{1} H\right)}{m_{1} H}+\frac{S_{1} \sinh \left(m_{2} H\right)}{m_{2} H}\right] .
$$

where

$$
m_{1}=m_{1}^{*} d=\sqrt{\frac{m^{2}}{2}\left(1+\sqrt{1-\frac{4}{m^{2} D a}}\right)}, \quad D a=\frac{k_{0}}{d^{2}}
$$




$$
m_{2}=m_{2}^{*} d=\sqrt{\frac{m^{2}}{2}\left(1-\sqrt{1-\frac{4}{m^{2} D a}}\right)}, \quad m=d\left(\mu / \eta^{\prime}\right)^{1 / 2},
$$

$m$ is the couple stress parameter and $D a$ is the permeability parameter (or Darcy number).

Further, (2.11) becomes

$$
\frac{\partial^{2} C}{\partial \eta^{2}}-\frac{k_{1} d^{2}}{D} C=\frac{d^{2}}{\lambda D} u_{x} \frac{\partial C}{\partial \xi}
$$

Assuming that there is no absorption at the walls, the boundary conditions for the concentration $C$ are

$$
\frac{\partial C}{\partial \eta}=0 \quad \text { for } \quad \eta= \pm H= \pm[1+\epsilon \sin (2 \pi \xi)]
$$

where $\epsilon=a / d$ is the amplitude ratio.

Assuming that $\partial C / \partial \xi$ is independent of $\eta$ at any cross-section and solving (2.14) under the boundary conditions (2.15), the solution for the concentration of the solute $C$ is given as

$$
\begin{aligned}
C(\eta)= & A \cosh (\alpha \eta)-\frac{d^{2}}{\lambda D} \frac{\partial C}{\partial \xi} \frac{k_{0}}{\mu} \frac{\partial p}{\partial x}\left\{\frac{S_{2}}{m_{1}^{2}-\alpha^{2}} \cosh \left(m_{1} \eta\right)-\frac{S_{1}}{m_{2}^{2}-\alpha^{2}} \cosh \left(m_{2} \eta\right)\right. \\
& \left.+\frac{S_{2}}{\alpha^{2} m_{1} H} \sinh \left(m_{1} H\right)-\frac{S_{1}}{\alpha^{2} m_{2} H} \sinh \left(m_{2} H\right)\right\}
\end{aligned}
$$

where

$$
A=\frac{d^{2}}{\lambda D} \frac{\partial C}{\partial \xi} \frac{k_{0}}{\mu} \frac{\partial p}{\partial x} \frac{1}{L}\left[\frac{m_{1} S_{2}}{m_{1}^{2}-\alpha^{2}} \sinh \left(m_{1} H\right)-\frac{m_{2} S_{1}}{m_{2}^{2}-\alpha^{2}} \sinh \left(m_{2} H\right)\right],
$$

$\alpha=d\left(k_{1} / D\right)^{1 / 2}$ and $L=\alpha \sinh (\alpha H)$.

The volumetric rate $Q$ at which the solute is transported across a section of the channel of unit breadth is defined by

$$
Q=\int_{-H}^{+H} C u_{x} d \eta
$$

Substituting (2.16) and (2.13) in (2.18), we get the volumetric rate $Q$ as

$$
Q=-\frac{2 d^{6}}{\lambda \mu^{2} D} \frac{\partial C}{\partial \xi}\left(\frac{\partial p}{\partial x}\right)^{2} F(\xi, \epsilon, \alpha, D a, m)
$$

where

$$
\begin{aligned}
F(\xi, \epsilon, \alpha, D a, m)= & (D a)^{2}\left\{\frac{S_{2}}{L\left(m_{1}^{2}-\alpha^{2}\right)}\left(\frac{m_{1} S_{2} \sinh \left(m_{1} H\right)}{m_{1}^{2}-\alpha^{2}}-\frac{m_{2} S_{1} \sinh \left(m_{2} H\right)}{m_{2}^{2}-\alpha^{2}}\right) B_{1}\right. \\
& +\frac{S_{1}}{L\left(m_{2}^{2}-\alpha^{2}\right)}\left(\frac{m_{2} S_{1} \sinh \left(m_{2} H\right)}{m_{2}^{2}-\alpha^{2}}-\frac{m_{1} S_{2} \sinh \left(m_{1} H\right)}{m_{1}^{2}-\alpha^{2}}\right) B_{2} \\
& +\frac{S_{1} S_{2}}{\alpha^{2} H} \sinh \left(m_{1} H\right) \sinh \left(m_{2} H\right)\left(\frac{m_{1}}{m_{2}\left(m_{1}^{2}-\alpha^{2}\right)}+\frac{m_{2}}{m_{1}\left(m_{2}^{2}-\alpha^{2}\right)}\right)
\end{aligned}
$$




$$
\begin{gathered}
\quad-\frac{S_{2}^{2} \sinh ^{2}\left(m_{1} H\right)}{\alpha^{2} H\left(m_{1}^{2}-\alpha^{2}\right)}-\frac{S_{1}^{2} \sinh ^{2}\left(m_{2} H\right)}{\alpha^{2} H\left(m_{2}^{2}-\alpha^{2}\right)}-\frac{S_{2}^{2}}{m_{1}^{2}-\alpha^{2}}\left(H+\frac{\sinh \left(2 m_{1} H\right)}{2 m_{1}}\right) \\
+\frac{S_{2}^{2} \sinh ^{2}\left(m_{1} H\right)}{m_{1}^{2} H\left(m_{1}^{2}-\alpha^{2}\right)}+\frac{S_{1} S_{2}}{m_{1}^{2}-\alpha^{2}}\left(\frac{1}{m_{1}^{2}-\alpha^{2}}+\frac{1}{m_{2}^{2}-\alpha^{2}}\right) B_{3} \\
\quad-\frac{S_{1} S_{2}}{m_{1} m_{2} H} \sinh \left(m_{1} H\right) \sinh \left(m_{2} H\right)\left(\frac{1}{m_{1}^{2}-\alpha^{2}}+\frac{1}{m_{2}^{2}-\alpha^{2}}\right) \\
\left.\quad-\frac{S_{1}^{2}}{m_{2}^{2}-\alpha^{2}}\left(H+\frac{\sinh \left(2 m_{2} H\right)}{2 m_{2}}\right)+\frac{S_{1}^{2} \sinh ^{2}\left(m_{2} H\right)}{m_{2}^{2} H\left(m_{2}^{2}-\alpha^{2}\right)}\right\}, \\
B_{1}=m_{1} \cosh (\alpha H) \sinh \left(m_{1} H\right)-\alpha \cosh \left(m_{1} H\right) \sinh (\alpha H), \\
B_{2}=m_{2} \cosh (\alpha H) \sinh \left(m_{2} H\right)-\alpha \cosh \left(m_{2} H\right) \sinh (\alpha H),
\end{gathered}
$$

and

$$
B_{3}=m_{1} \cosh \left(m_{2} H\right) \sinh \left(m_{1} H\right)-m_{2} \cosh \left(m_{1} H\right) \sinh \left(m_{2} H\right) .
$$

Comparing (2.19) with Fick's law of diffusion, we find that the solute is dispersed relative to a plane moving with the mean speed of the flow with an effective dispersion coefficient $D^{*}$ given by

$$
D^{*}=2 \frac{d^{6}}{\mu^{2} D}\left(\frac{\partial p}{\partial x}\right)^{2} F(\xi, \epsilon, \alpha, D a, m) .
$$

Let the average of $F$ be $\bar{F}$ and is defined by

$$
\bar{F}=\int_{0}^{1} F(\xi, \epsilon, \alpha, D a, m) d \xi .
$$

\subsection{Diffusion with combined homogeneous and heterogeneous chemical reactions}

We now discuss the problem of diffusion with a first-order irreversible chemical reaction taking place both in the bulk of the medium (homogeneous) as well as at the walls (heterogeneous) of the channel which are assumed to be catalytic to chemical reaction. The simplified diffusion equation is same as (2.11), i.e.,

$$
\frac{\partial C}{\partial t}+u \frac{\partial C}{\partial x}=D \frac{\partial^{2} C}{\partial y^{2}}-k_{1} C
$$

The differential material balance at the walls (Philip and Peeyush [20]) gives the boundary conditions

$$
\frac{\partial C}{\partial y}+f C=0 \text { at } y=h=\left[d+a \sin \frac{2 \pi}{\lambda}(x-\bar{u} t)\right]
$$




$$
\frac{\partial C}{\partial y}-f C=0 \text { at } y=-h=-\left[d+a \sin \frac{2 \pi}{\lambda}(x-\bar{u} t)\right] .
$$

If we introduce the dimensionless variables (2.12) and assume the limiting condition of Taylor [32, 33, 34], the diffusion equation remains as (2.14) but subject to the boundary conditions

$$
\begin{aligned}
& \frac{\partial C}{\partial \eta}+\beta C=0 \text { for } \eta=H=[1+\epsilon \sin (2 \pi \xi)] \\
& \frac{\partial C}{\partial \eta}-\beta C=0 \text { for } \eta=-H=-[1+\epsilon \sin (2 \pi \xi)]
\end{aligned}
$$

where $\beta=f d$ is the heterogeneous reaction rate parameter corresponding to catalytic reaction at the walls.

The solution of (2.14) satisfying the boundary conditions (2.25) and (2.26) is

$$
\begin{aligned}
C(\eta)= & A^{\prime} \cosh (\alpha \eta)+\frac{d^{2}}{\lambda D} \frac{\partial C}{\partial \xi} \frac{k_{0}}{\mu} \frac{\partial p}{\partial x}\left[\frac{S_{2}}{m_{1}^{2}-\alpha^{2}} \cosh \left(m_{1} \eta\right)\right. \\
& \left.-\frac{S_{1}}{m_{2}^{2}-\alpha^{2}} \cosh \left(m_{2} \eta\right)+\frac{S_{2}}{\alpha^{2} m_{1} H} \sinh \left(m_{1} H\right)-\frac{S_{1}}{\alpha^{2} m_{2} H} \sinh \left(m_{2} H\right)\right]
\end{aligned}
$$

where

$$
\begin{aligned}
A^{\prime}= & \frac{d^{2}}{\lambda D} \frac{\partial C}{\partial \xi} \frac{k_{0}}{\mu} \frac{\partial p}{\partial x} \frac{1}{L^{\prime}}\left[\frac{m_{1} S_{2}}{m_{1}^{2}-\alpha^{2}} \sinh \left(m_{1} H\right)-\frac{m_{2} S_{1}}{m_{2}^{2}-\alpha^{2}} \sinh \left(m_{2} H\right)\right. \\
& +\frac{\beta S_{2}}{m_{1}^{2}-\alpha^{2}} \cosh \left(m_{1} H\right)-\frac{\beta S_{1}}{m_{2}^{2}-\alpha^{2}} \cosh \left(m_{2} H\right)+\frac{\beta S_{2}}{\alpha^{2} m_{1} H} \sinh \left(m_{1} H\right) \\
& \left.-\frac{\beta S_{1}}{\alpha^{2} m_{2} H} \sinh \left(m_{2} H\right)\right]
\end{aligned}
$$

and $L^{\prime}=\alpha \sinh (\alpha H)+\beta \cosh (\alpha H)$.

Substituting (2.27) and (2.13) in (2.18), we get

$$
Q=-2 \frac{d^{6}}{\lambda \mu^{2} D} \frac{\partial C}{\partial \xi}\left(\frac{\partial p}{\partial x}\right)^{2} G(\xi, \epsilon, \alpha, \beta, D a, m)
$$

where

$$
\begin{aligned}
G(\xi, \epsilon, \alpha, \beta, D a, m)= & (D a)^{2}\left\{\frac{S_{2}}{L^{\prime}\left(m_{1}^{2}-\alpha^{2}\right)}\left(\frac{m_{1} S_{2} \sinh \left(m_{1} H\right)}{m_{1}^{2}-\alpha^{2}}-\frac{m_{2} S_{1} \sinh \left(m_{2} H\right)}{m_{2}^{2}-\alpha^{2}}\right) B_{1}\right. \\
& +\frac{S_{1}}{L^{\prime}\left(m_{2}^{2}-\alpha^{2}\right)}\left(\frac{m_{2} S_{1} \sinh \left(m_{2} H\right)}{m_{2}^{2}-\alpha^{2}}-\frac{m_{1} S_{2} \sinh \left(m_{1} H\right)}{m_{1}^{2}-\alpha^{2}}\right) B_{2} \\
& -\frac{S_{2}^{2} \sinh ^{2}\left(m_{1} H\right) \sinh (\alpha H)}{\alpha H L^{\prime}\left(m_{1}^{2}-\alpha^{2}\right)}-\frac{S_{1}^{2} \sinh ^{2}\left(m_{2} H\right) \sinh (\alpha H)}{\alpha H L^{\prime}\left(m_{2}^{2}-\alpha^{2}\right)}
\end{aligned}
$$




$$
\begin{aligned}
& +\frac{S_{1} S_{2}}{\alpha H L^{\prime}}\left(\frac{m_{1}}{m_{2}\left(m_{1}^{2}-\alpha^{2}\right)}+\frac{m_{2}}{m_{1}\left(m_{2}^{2}-\alpha^{2}\right)}\right) \sinh \left(m_{1} H\right) \sinh \left(m_{2} H\right) \sinh (\alpha H) \\
& +\frac{\beta S_{2}}{L^{\prime}\left(m_{1}^{2}-\alpha^{2}\right)}\left(\frac{S_{2} \cosh \left(m_{1} H\right)}{m_{1}^{2}-\alpha^{2}}-\frac{S_{1} \cosh \left(m_{2} H\right)}{m_{2}^{2}-\alpha^{2}}\right) B_{1} \\
& +\frac{\beta S_{1}}{L^{\prime}\left(m_{2}^{2}-\alpha^{2}\right)}\left(\frac{S_{1} \cosh \left(m_{2} H\right)}{m_{2}^{2}-\alpha^{2}}-\frac{S_{2} \cosh \left(m_{1} H\right)}{m_{1}^{2}-\alpha^{2}}\right) B_{2} \\
& +\frac{\beta S_{2} \cosh \left(m_{1} H\right) \sinh (\alpha H)}{\alpha H L^{\prime}\left(m_{1}^{2}-\alpha^{2}\right)}\left(\frac{S_{1} \sinh \left(m_{2} H\right)}{m_{2}}-\frac{S_{2} \sinh \left(m_{1} H\right)}{m_{1}}\right) \\
& +\frac{\beta S_{1} \cosh \left(m_{2} H\right) \sinh (\alpha H)}{\alpha H L^{\prime}\left(m_{2}^{2}-\alpha^{2}\right)}\left(\frac{S_{2} \sinh \left(m_{1} H\right)}{m_{1}}-\frac{S_{1} \sinh \left(m_{2} H\right)}{m_{2}}\right) \\
& +\frac{\beta S_{2}}{\alpha^{2} H L^{\prime}\left(m_{1}^{2}-\alpha^{2}\right)}\left(\frac{S_{2} \sinh \left(m_{1} H\right)}{m_{1}}-\frac{S_{1} \sinh \left(m_{2} H\right)}{m_{2}}\right) B_{1} \\
& +\frac{\beta S_{1}}{\alpha^{2} H L^{\prime}\left(m_{2}^{2}-\alpha^{2}\right)}\left(\frac{S_{1} \sinh \left(m_{2} H\right)}{m_{2}}-\frac{S_{2} \sinh \left(m_{1} H\right)}{m_{1}}\right) B_{2} \\
& +\frac{\beta S_{2} \sinh \left(m_{1} H\right) \sinh (\alpha H)}{\alpha^{3} m_{1} H^{2} L^{\prime}}\left(\frac{S_{1} \sinh \left(m_{2} H\right)}{m_{2}}-\frac{S_{2} \sinh \left(m_{1} H\right)}{m_{1}}\right) \\
& +\frac{\beta S_{1} \sinh \left(m_{2} H\right) \sinh (\alpha H)}{\alpha^{3} m_{2} H^{2} L^{\prime}}\left(\frac{S_{2} \sinh \left(m_{1} H\right)}{m_{1}}-\frac{S_{1} \sinh \left(m_{2} H\right)}{m_{2}}\right) \\
& -\frac{S_{2}^{2}}{m_{1}^{2}-\alpha^{2}}\left(H+\frac{\sinh \left(2 m_{1} H\right)}{2 m_{1}}\right)+\frac{S_{1} S_{2}}{m_{1}^{2}-\alpha^{2}}\left(\frac{1}{m_{1}^{2}-\alpha^{2}}+\frac{1}{m_{2}^{2}-\alpha^{2}}\right) B_{3} \\
& +\frac{S_{2}^{2} \sinh ^{2}\left(m_{1} H\right)}{m_{1}^{2} H\left(m_{1}^{2}-\alpha^{2}\right)}-\frac{S_{1} S_{2}}{m_{1} m_{2} H} \sinh \left(m_{1} H\right) \sinh \left(m_{2} H\right)\left(\frac{1}{m_{1}^{2}-\alpha^{2}}+\frac{1}{m_{2}^{2}-\alpha^{2}}\right) \\
& \left.-\frac{S_{1}^{2}}{m_{2}^{2}-\alpha^{2}}\left(H+\frac{\sinh \left(2 m_{2} H\right)}{2 m_{2}}\right)+\frac{S_{1}^{2} \sinh ^{2}\left(m_{2} H\right)}{m_{2}^{2} H\left(m_{2}^{2}-\alpha^{2}\right)}\right\} \text {. }
\end{aligned}
$$

Comparing (2.29) with Fick's Law of Diffusion, we find that the solute is dispersed relative to a plane moving with the mean speed of the flow with an effective dispersion coefficient $D^{*}$ given by

$$
D^{*}=2 \frac{d^{6}}{\mu^{2} D}\left(\frac{\partial p}{\partial x}\right)^{2} G(\xi, \epsilon, \alpha, \beta, D a, m) .
$$

The average of $G$ denoted by $\bar{G}$ is defined as

$$
\bar{G}=\int_{0}^{1} G(\xi, \epsilon, \alpha, \beta, D a, m) d \xi .
$$

\section{Results and discussion}

As given in (2.22)and (2.32), the expressions for $\bar{F}$ and $\bar{G}$ have been obtained by numerical integration using MATHEMATICA software. The effects of various parameters on the average 


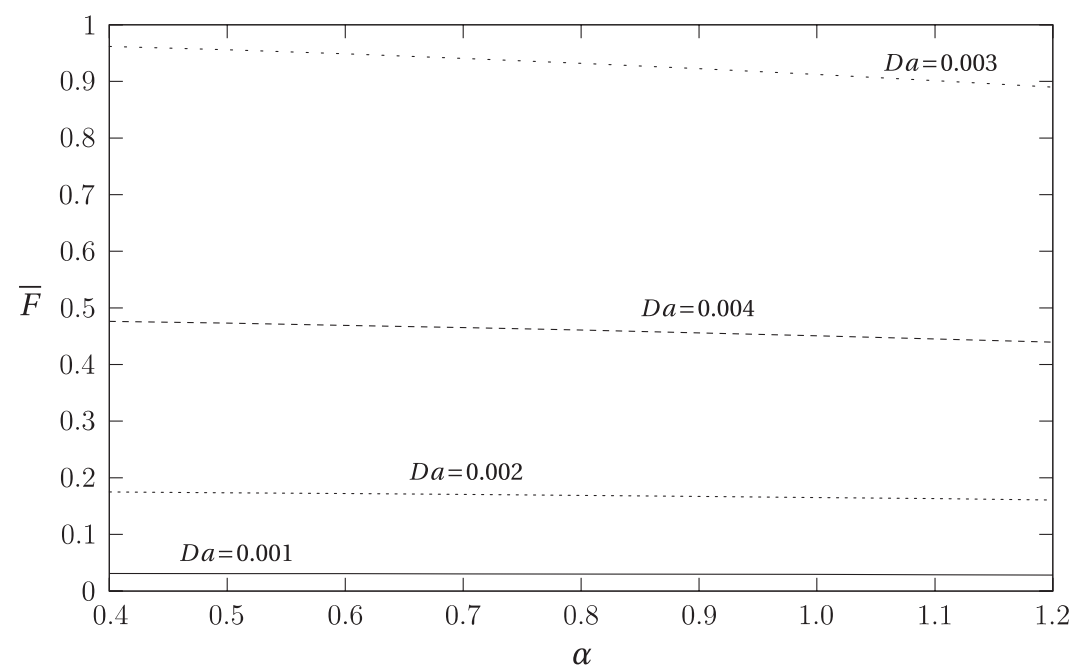

Figure 2: Effect of $D a$ on $\bar{F}$ for $m=2.0$ and $\epsilon=0.2$.

effective dispersion coefficient can be observed through the functions $\bar{F}(\xi, \epsilon, \alpha, \beta, D a, m$ ) (for homogeneous case) and $\bar{G}(\xi, \epsilon, \alpha, \beta, D a, m$ ) (for combined homogeneous and heterogeneous case). The functions $\bar{F}$ and $\bar{G}$ have been numerically evaluated for different values of relevant parameters and presented graphically. The important parameters involved in the expressions are: the amplitude ratio $\epsilon$, the homogeneous reaction parameter $\alpha$, the heterogeneous reaction rate parameter $\beta$, the permeability parameter (or Darcy number) $D a$, and couple stress parameter $m$.

\section{Homogeneous chemical reaction}

Figs. 2-4 show that average effective dispersion coefficient $\bar{F}$ decreases with homogeneous reaction rate parameter $\alpha$. This implies that homogeneous chemical reaction tends to decrease the dispersion of the solute. This result is expected since increase in $\alpha$ leads to increasing number of moles of solute undergoing chemical reaction, which results in the decrease of dispersion. The result that dispersion decreases with $\alpha$ agrees with previous results obtained by Gupta and Gupta [10], Dutta et al.[7], Ramana Rao and Padma [24, 25], Padma and Ramana Rao [19], Shukla et al. [27]. Further, average dispersion increases with permeability parameter (or Darcy number) $D a$ (Fig.2) but decreases with amplitude ratio $\epsilon$ (Fig.3) and couple stress parameter $m$ (Fig. 4). The result that dispersion decreases with couple stress parameter $m$ agrees with the result obtained by Soundalgekar and Chaturani [29]. 


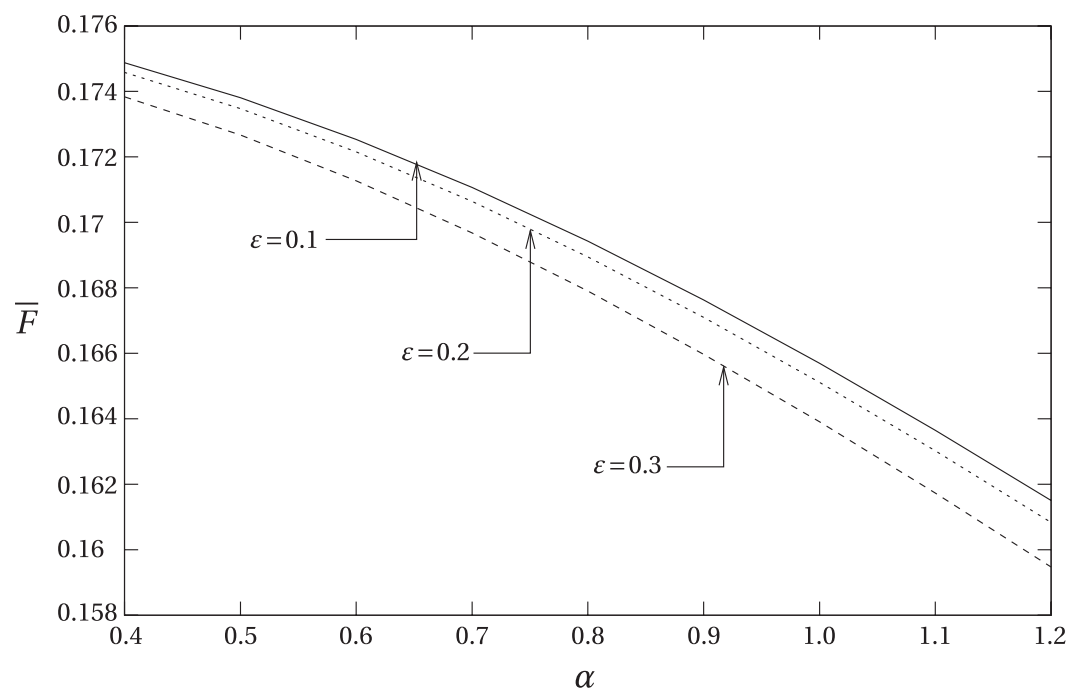

Figure 3: Effect of $\epsilon$ on $\bar{F}$ for $m=2.0$ and $D a=0.002$.

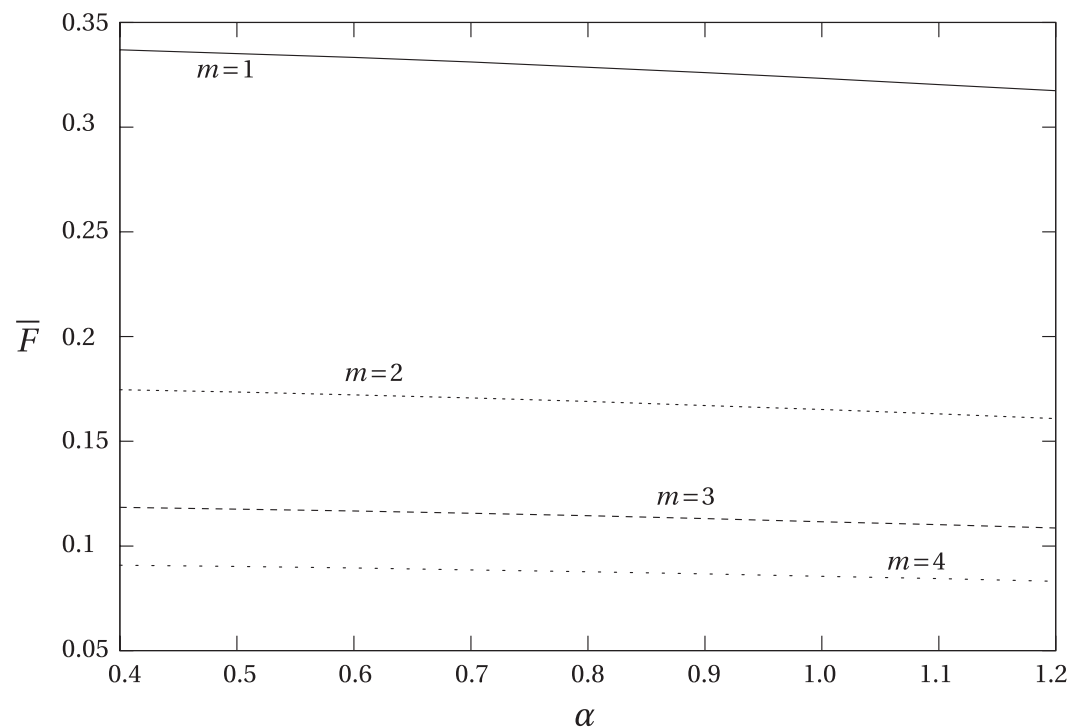

Figure 4: Effect of $m$ on $\bar{F}$ for $\epsilon=0.2$ and $D a=0.002$.

\section{Combined homogeneous and heterogeneous chemical reactions}

Figs. 5-8 show the effects of various parameters on the average dispersion coefficient $\bar{G}$ for the case of combined first order chemical reactions both in the bulk and at the walls. Average dispersion coefficient $\bar{G}$ increases with permeability parameter $D a$ (Fig.5). But $\bar{G}$ decreases with homogeneous chemical reaction parameter $\alpha$ (Fig.6), couple stress parameter $m$ (Fig.7) and amplitude ratio $\epsilon$ (Fig.8). Further, Figs. 5-8 reveal that dispersion decreases 


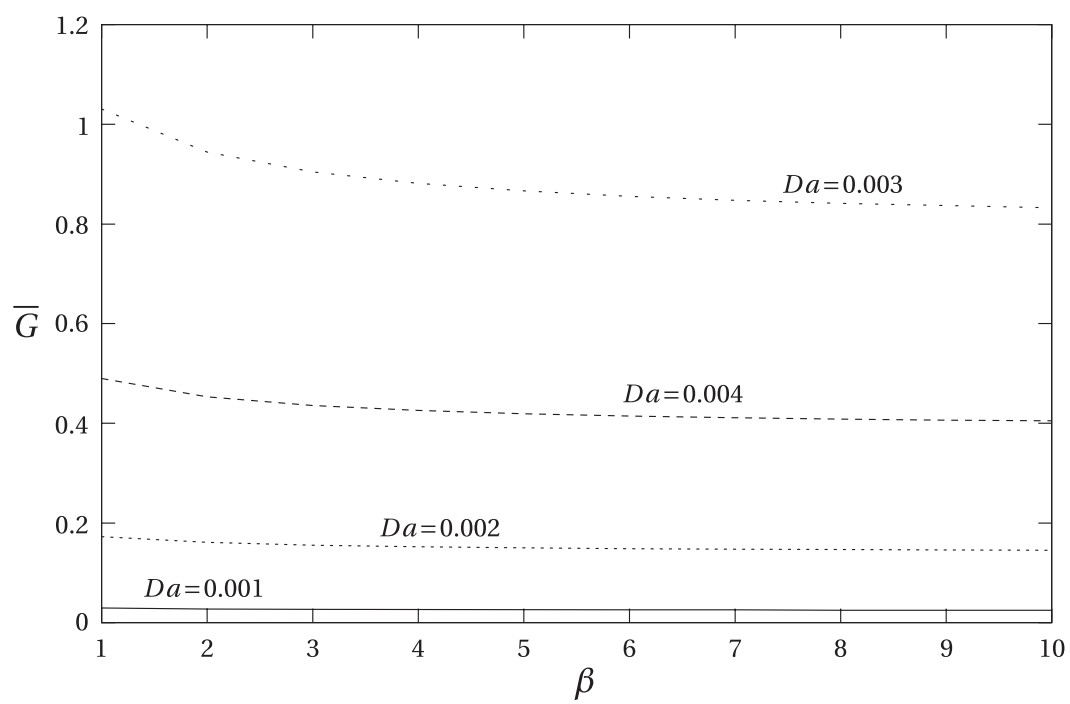

Figure 5: Effect of $D a$ on $\bar{G}$ for $\alpha=1.0, m=2.0$ and $\epsilon=0.2$.

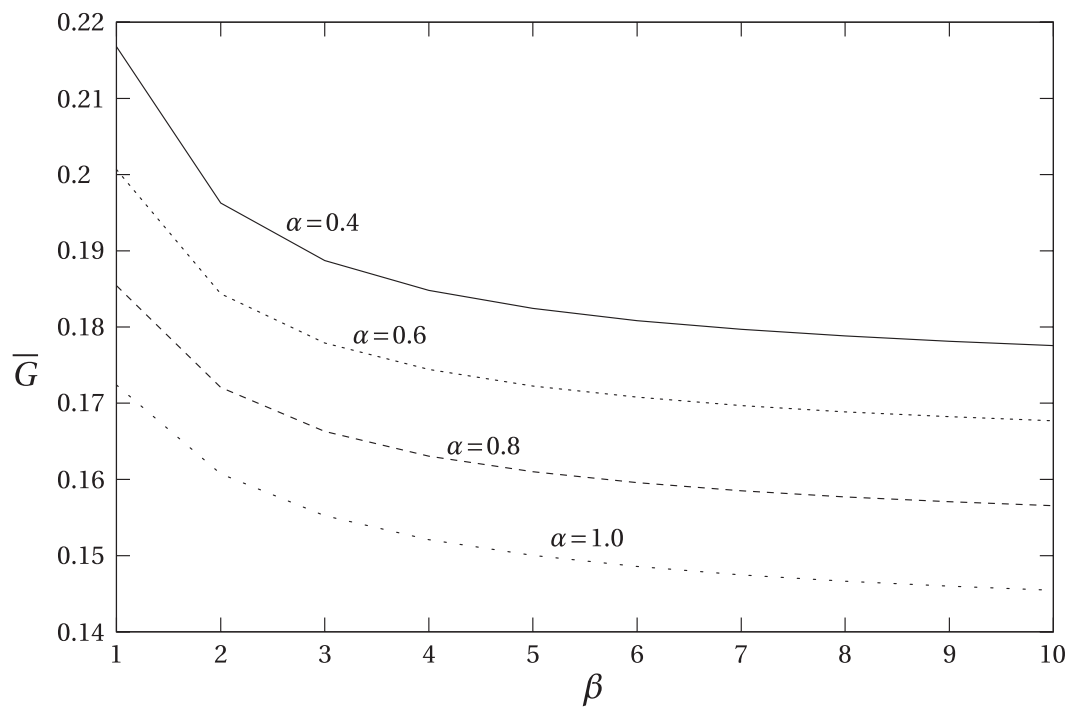

Figure 6: Effect of $\alpha$ on $\bar{G}$ for $m=2.0, D a=0.002$ and $\epsilon=0.2$.

with heterogeneous reaction parameter $\beta$. The result that dispersion decreases with heterogeneous reaction rate parameter $\beta$ agrees with previous results obtained by Gupta and Gupta [10], Ramana Rao and Padma [24, 25], Padma and Ramana Rao [19]. This implies that heterogeneous chemical reaction tends to decrease the dispersion of the solute. 


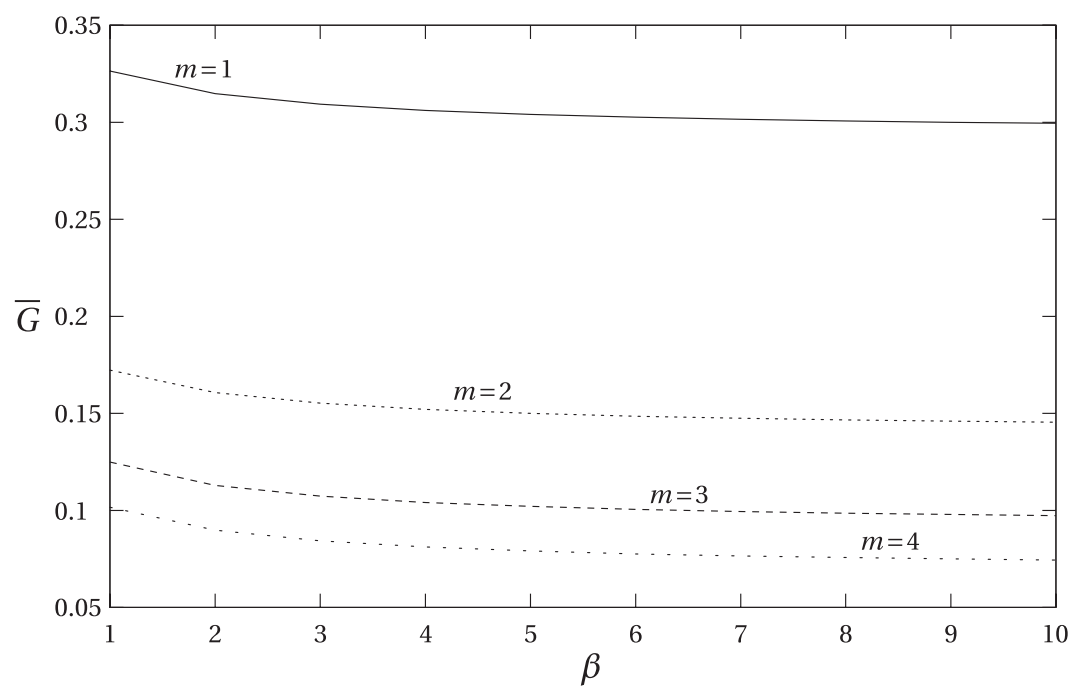

Figure 7: Effect of $m$ on $\bar{G}$ for $\alpha=1.0, D a=0.002$ and $\epsilon=0.2$.

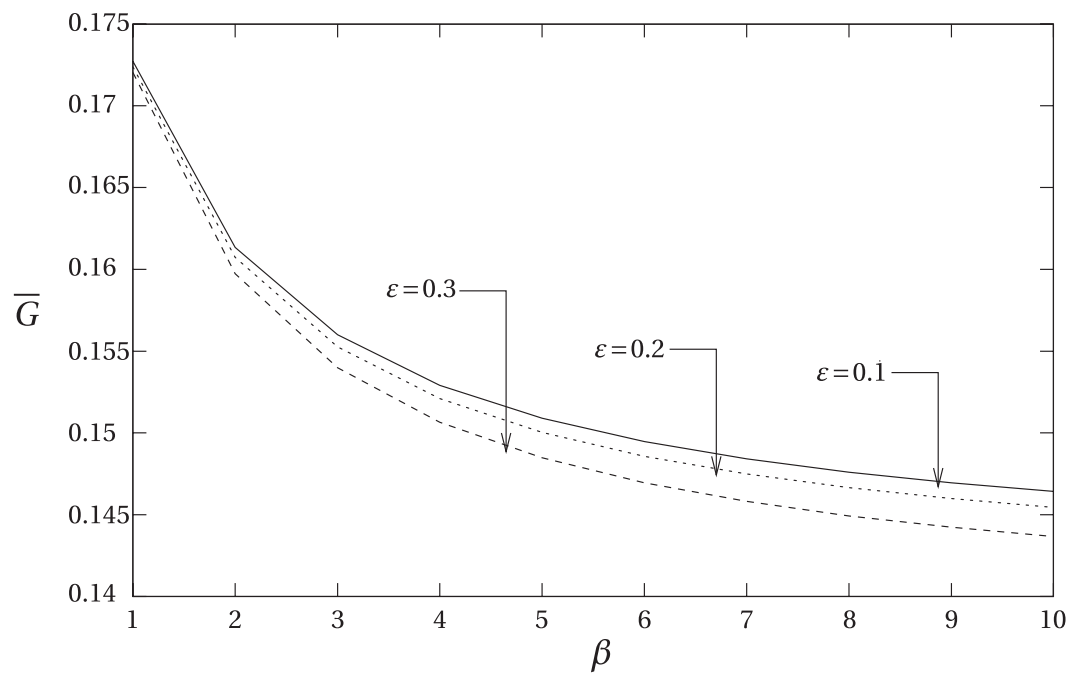

Figure 8: Effect of $\epsilon$ on $\bar{G}$ for $m=2.0, D a=0.002$ and $\alpha=1.0$.

\section{Conclusion}

The dispersion of a solute in peristaltic motion of a couple stress fluid through a porous medium with both homogeneous and heterogeneous chemical reactions has been studied under long wavelength approximation and Taylor's limiting condition. It is observed that average effective coefficient of dispersion increases with permeability parameter. But it decreases with homogeneous chemical reaction rate parameter $\alpha$, couple stress parameter $m$, amplitude ratio $\epsilon$ and heterogeneous chemical reaction rate parameter $\beta$. 


\section{References}

[1] S. Abbasbandy, T. Hayat, R. Ellahi and S. Asghar, Numerical results of flow in a third grade fluid between two porous walls, Zeitschrift Fur Naturforschung A, 64(a) (2009), 59-64.

[2] M. A. Al-Nimr and M. K. Alkam, Basic fluid flow problems in porous media, Journal of Porous Media, 3(2000), 45-59.

[3] R. Aris, On the dispersion of a solute in a fluid flowing through a tube, Proc. Roy. Soc. Lond. A235(1956), 67-77.

[4] S. Ashgar, C. Khalique and R. Ellahi, Influence of a Partial Slip on Flows of a Second Grade Fluid in Porous Medium, Journal of Porous Media, 10(2007), 797-805.

[5] P. Chandra and R. P. Agarwal, Dispersion in simple microfluid flows, International Journal of Engineering Science, 21(1983), 431-442.

[6] Dulal Pal, Effect of chemical reaction on the dispersion of a solute in a porous medium, Applied Mathematical Modeling, 23(1999), 557-566.

[7] B. K. N. Dutta, C. Roy and A. S. Gupta, Dispersion of a solute in a non-Newtonian fluid with simultaneous chemical reaction, Mathematica-Mechanica fasc., 2(1974), 78-82.

[8] R. Ellahi and S. Afzal, Effects of variable viscosity in a third grade fluid with porous medium: An analytical solution, Communication in Nonlinear Science and Numerical Simulations, 14, (2009), 2056-2072.

[9] Y. C. Fung and C. S. Yih, Peristaltic transport, J. Appl. Mech. Trans. ASME., 5(1968), 669-675.

[10] P. S. Gupta and A. S. Gupta, Effect of homogeneous and heterogeneous reactions on the dispersion of a solute in the laminar flow between two plates, Proc. Roy. Soc. Lond. A330(1972), 59-63.

[11] T. Hayat, M. Khan, S. Ashgar and A. M. Siddiqui, A mathematical model of peristalsis in tubes through a porous medium, Journal of Porous Media, 9(2006), 55-67.

[12] S. Islam and C. Y. Zhou, Exact solutions for two dimensional flows of couple stress fluids, Z. angew. Math. Phys., 58(2007), 1035-1048.

[13] I. Khan and R. Ellahi, Some MHD flows of a second grade fluid through the porous medium, Journal of Porous Media, 11 (2008), 389-400.

[14] M. Khan and R. Ellahi, Exact solution for oscillatory flows of generalized Oldroyd-B fluid through porous medium in a rotating frame, Journal of Porous Media, 12(2009),777-788.

[15] K. N. Mehta and M. C. Tiwari, Dispersion in presence of slip and chemical reactions in porous wall tube flow, Def Sci J., 38(1988), 1-11.

[16] Kh. S. Mekheimer and Y. Abd Elmaboud, Peristaltic flow of a couple stress fluid in an annulus: Application of an endoscope, Physica A., 387(2008), 2403-2415.

[17] J. C. Misra and S. K. Pandey, Peristaltic flow of a multilayered power-law fluid through a cylindrical tube, International Journal of Engineering Science, 39(2001), 387-402.

[18] J. C. Misra and S. K.Ghosh, A mathematical model for the study of blood flow through a channel with permeable walls, Acta Mechanica, 122(1997), 137-153.

[19] D. Padma and V. V. Ramana Rao, Effect of Homogeneous and heterogeneous reaction on the dispersion of a solute in laminar flow between two parallel porous plates, Indian Journal of Technology, 14(1976), 410-412.

[20] D. Philip and P. Chandra, Effects of heterogeneous and homogeneous reactions on the dispersion of a solute in simple microfluid, Indian Journal of Pure Applied Mathematics, 24(1993), 551-561.

[21] G. Radhakrishnamacharya, Some flow problems in bio-fluid dynamics, Ph.D. Thesis. IIT Kharagpur (1978).

[22] G. Radhakrishnamacharya, Long wavelength approximation to peristaltic motion of a power law fluid, Rheologica Acta, 21(1982), 30-35.

[23] A. Ramachandra Rao and M. Mishra, Peristaltic transport of a power-law fluid in a porous tube, Journal of Non-Newtonian Fluid Mechanics, 121(2004), 163-174.

[24] V. V. Ramana Rao, and D. Padma, Homogeneous and heterogeneous reaction on the dispersion of a solute in MHD Couette flow, Curr. Sci., 44(1975), 803-804.

[25] V. V. Ramana Rao, and D. Padma, Homogeneous and heterogeneous reaction on the dispersion of a solute in MHD Couette flow II, Curr. Sci., 46(1977), 42-43. 
[26] A. H. Shapiro, M. Y. Jaffrin and S. L. Weinberg, Peristaltic pumping with long wavelengths at low Reynold number, J. Fluid Mech., 37(1969), 799-825.

[27] J. B. Shukla, R. S. Parihar and B. R. P. Rao, Dispersion in non-Newtonian fluids: Effects of chemical reaction, Rheologica Acta, 18(1979), 740-748.

[28] A. M. Sobh, Interaction of couple stresses and slip flow on peristaltic transport in uniform and nonuniform channels, Turkish J. Eng. Env. Sci. 32(2008), 117-123.

[29] V. M. Soundalgekar, and P. Chaturani, Effects of couple-stresses on the dispersion of a soluble matter in a pipe flow of blood, Rheologica Acta, 19(1980), 710-715.

[30] L. M. Srivastava, Peristaltic transport of a couple-stress fluid, Rheologica Acta, 25(1986), 638-641.

[31] V.K. Stokes, Couple stress fluid, Physics in Fluids, 9(1966), 1709-1715.

[32] G. I. Taylor, Dispersion of soluble matter in solvent flowing slowly through a tube, Proc. Roy. Soc. Lond. A219(1953), 186-203.

[33] G. I. Taylor, The dispersion of matter in turbulent flow through a pipe, Proc. Roy. Soc. Lond. A223(1954a), 446-468.

[34] G. I. Taylor, Conditions under which dispersion of a solute in a stream of solvent can be used to measure molecular diffusion, Proc. Roy. Soc. Lond. A225(1954b), 47-3-477.

Department of Mathematics, National Institute of Technology, Warangal-506 004, A.P., India.

E-mail: grk.nitw@yahoo.com 